\title{
HENRI BERGSON Y GEORGE BERNARD SHAW: HÁBITO, VIDA, MUERTE
}

\author{
ÁLVARO CORTINA URDAMPILLETA \\ Universidad Diego Portales
}

\begin{abstract}
RESUMEN: Este artículo indaga en algunos aspectos de la antropología filosófica expuesta en la obra del francés Henri Bergson. Para ello, nos serviremos de la confrontación de su doctrina original con la de un bergsoniano sui generis, el intelectual y dramaturgo irlandés George Bernard Shaw. El Prefacio. A medio siglo infiel, de Shaw, incluido en su obra más ambiciosa, Vuelta a Matusalén. Un Pentateuco Metabiológico, de 1921, será el texto base con el que haremos dialogar a Bergson con este periférico bergsonismo. Tomaremos como hilo conductor la teoría sobre el hábito, en el contexto de la metafísica de la evolución de los seres orgánicos, y lo llevaremos hasta el concepto límite, que parece conciliar ambas posturas: la idea de muerte superable. Ni en Bergson ni en Shaw los hábitos (la Cultura) forman parte integral de la esencia del hombre, y la muerte (cuestión en la que Bergson es mucho más elusivo) juega en ellos un rol anfibio, entre Cultura y Naturaleza.
\end{abstract}

PALABRAS CLAVE: Henri Bergson, George Bernard Shaw, neo-lamarckismo, neodarwinismo, evolución creadora, hábito, muerte.

\section{Henri Bergson and George Bernard Shaw: Habit, Life, Death}

ABSTRACT: This article tries to illuminate certain aspects of the philosophical anthropology exposed in the works written by the french thinker Henri Bergson. We will analyze the confrontation of his original doctrine with the sui generis bergsonianism of the irish intellectual and playwright George Bernard Shaw. The Shaw's Preface. An Infidel Half Century, included in Back to Methuselah, written in 1921, will be the main text that we will use to engage Henri Bergson with this peripheral bergsonianism. The theories about the concept of habit will be our guideline, in the context of the metaphysics of the organic evolution, and we will reach to the 'limiting concept' of death, which seems to reconcile both positions: the surmountable death. In Bergson and in Shaw habits (the Culture) are not an integral part of the essence of the human being, and death (question in which Bergson is much more elusive) plays in their work an amphibious role, between Culture and Nature.

KEY WORDS: Henri Bergson, George Bernard Shaw, neo-lamarckism, neo-darwinism, creative evolution, habit, death.

Con la fuente bibliográfica de Floris Delattre ${ }^{1}$, los biógrafos Philippe Soulez y Frédéric Worms señalan un boom del filósofo francés Henri Bergson en Inglaterra entre 1910 y el estallido de la Segunda Guerra Mundial, en 1914². En el capítulo VI de la monografía de los dos autores, intitulado La réception de Bergson en Grande. Bretagne et aux ÉtatsUnis valoran los apoyos y relaciones intelectuales que el filósofo francés tenía entre los británicos. Soulez y Worms no pierden la oportunidad de mentar, sólo por encima, una picante situación, una famosa cena ${ }^{3}$, en 1911, en Londres, a la que acude el ya por entonces muy célebre autor de La evolución creadora, en la que intervino el polemista, dramaturgo, bergsoniano sui generis George Bernard Shaw, firma también en auge en teatros y ágoras, desde los primeros años del siglo xx. Pero la fuente que usan Soulez y Worms, no

Delattre, F., "Henri Bergson et l'Anglaterre», in Les études bergsoniennes. Vol. II, PUF, 1949.

2 Soulez, P., Worms, F. Bergson. Biographie. PUF, Paris, 2002, p. 119.

3 Ibid., p. 123. 
es en este caso Floris Delattre, sino un testigo que escribió sobre el suceso mucho tiempo después, en 1956. Hablamos de Lord Bertrand Russell (quien adoptó, por cierto, una posición contraria a aquella nueva metafísica, à la Julien Benda, en la isla). Por estas periferias, los extrarradios del bergsonismo, accederemos a algunos de sus temas capitales.

Para ofrecer una prueba de lo «anormalmente vanidoso» ${ }^{4}$ que era Shaw, Russell recreó dos acontecimientos que el irlandés protagonizó en su presencia. Nos interesa el primero de estos acontecimientos, una cena en Londres. En presencia del pensador francés, y rodeado de filósofos académicos «que consideraban a Bergson con más sentido crítico ${ }^{5}$, Russell explica que "Shaw empezó a exponer la filosofía de Bergson al estilo del prefacio de Matusalén. En su versión, era difícil que la filosofía bergsoniana obtuviera el respeto de los filósofos profesionales, y Bergson interrumpió, suavemente: 'No, no. No es exactamente eso'. Pero Shaw no se inmutó en absoluto, y respondió: 'Mi querido amigo, entiendo su filosofía mucho mejor que usted'. Bergson cerró los puños y estuvo a punto de estallar, pero, con mucho esfuerzo, consiguió dominarse y el monólogo expositivo de Shaw continuó» ${ }^{6}$. Según Russell, "la antipatía de Shaw hacia Darwin» sólo pudo hacer «de él un admirador de Bergson » ${ }^{7}$. Así aporta Russell la imagen de Shaw como homo loquax ${ }^{8}$, como nocivo intelectual palabrero que enturbia la doctrina de un filósofo. Aunque aquí Russell refiere el "estilo del prefacio de Matusalén», en un escrito anterior había dicho expresamente que la tetralogía Vuelta a Matusalén. Un Pentateuco metabiológico (1921), es «bergsonismo puro» ${ }^{9}$ ¿Es pues una irresponsable distorsión del «bergsonismo» o «bergsonismo puro»? ¿El enfrentamiento con el darwinismo le llevó a Shaw, legítimamente, aproximarse al neo-darwinismo? ¿Es, en definitiva, Bergson, un anti-darwinista en el mismo sentido en que éste es anti-darwinista?

Vuelta a Matusalén es un pentateuco, una pentalogía formada por las obras En el comienzo, El evangelio de los hermanos Barnabas, La cosa sucede, Tragedia de un hombre entrado en años y Tan lejos como el pensamiento pueda llegar de aire rapsódico, fáustico, con ilación "de tesis» y de difícil representación escénica. Abarca un trazo temporal que va desde el Génesis hasta más allá del año 3.000 después de Cristo. Una obra en torno a la abolición de la muerte y aún resentida por el pesar y el penar de la última guerra mundial (un tono similar, por cierto, a Las dos fuentes de la moral y de la religión, de Bergson, en 1932). El Prefacio. A medio siglo infiel, prólogo de Vuelta a Matusalén, característicamente más largo que ninguna de las cinco obras reunidas en el volumen, emplea repetidamente la expresión "Creative Evolution» (sin citar al autor de La evolución creativa, de 1907) y se autoinscribe dentro de un movimiento neo-lamarckista (lamarcko-shawiano) humanista. Se sitúa dentro de «aquello que está emergiendo, bajo el título de "Evolución Creadora”, como la genuina religión científica en la que todos los hombres sabios están ahora ansiosamente atentos ${ }^{10}$. Este prólogo, el Prefacio, continúa la senda de otro prefacio muy anterior de Shaw, de una obra que inaugura su etapa más ambiciosa, el Shaw

\footnotetext{
4 Russell, B., Retratos de memoria y otros ensayos, Trad. Manuel Suárez, M. Aguilar Editor, Buenos Aires, 1960, p. 76.

Ibid.

Ibid., pág.77.

Ibid.

8 Bergson, H., El pensamiento y lo moviente, Trad. Heliodoro García. Espasa, Madrid, 1971, p. 81.

9 Russell, B., Historia de la Filosofía Occidental. Trad. Julio Gómez de la Serna y Antonio Dorta. Austral, Madrid, 1999, p. 409.

${ }_{10}$ SHaw, G. B., Back to Methuselah. A metabiological Pentateuch. Bernhard Tauchnitz. Collection of british and american authors. Vol. 4578, 1922, p. 44.
} 
"filósofo» ${ }^{11}$, Hombre y superhombre, de $1905^{12}$. Entre este título de 1905 y el ya mentado, de 1921, se "constituye el tronco principal de su obra» ${ }^{13}$. En esta línea aparece, abiertamente mentada, la influencia de Nietzsche y de Schopenhauer, también la de Lamarck, pero dado que Hombre y superhombre es previo a la idea del élan o impulso vital bergsoniano, aparecida en La evolución creadora ${ }^{14}$, no me valdré tampoco de Hombre y superhombre, ni de su prefacio también jugoso y discursivo, Manual revolucionario. En todo caso, Vuelta a Matusalén y sus 49 epígrafes son la expresa recapitulación de esta etapa madura, desde 1905. Para el propio Shaw, Vuelta a Matusalén fue su principal aportación, la obra maestra de su etapa madura (el dramaturgo nació en 1856).

Como en otros países, Bergson tuvo un influjo importante, más allá de la academia. En Inglaterra mantuvo vínculo con profesores universitarios como Arthur James Balfour, Lord Richard Haldane, el teólogo Hastings Rashdall, James Sully o Herbert Wildon Carr. Se sabe de la recepción que sus célebres conferencias de 1911, en Oxford y Birmingham, o su curso (On nature of the soul) en el University College of London, así como su excelente relación con instituciones británicas (por ejemplo, fue doctor honoris causa por Cambridge, en 1920). Pero el «caso Shaw» nos deja entrever su vínculo directo, y la índole de tal vínculo, con una de las figuras mayores de las letras del periodo victoriano y eduardiano. Quien, según Chesterton, «ha mejorado las discusiones filosóficas haciéndolas más populares. Pero también ha mejorado las distracciones populares haciéndolas más filosóficas» ${ }^{15}$. Arthur Ganz explica en George Bernard Shaw: «sin duda pudo haber algún aspecto en el que el prestigio de Bergson tentó a Shaw para hacer la auténtica declaración que la "evolución creativa" era la "religión del siglo xx"16. Examinemos pues A medio siglo infiel a la luz de los textos de Henri Bergson, para calibrar el grado de esta influencia o la libre adopción de sus ideas, como si de volver a la cena antigua se tratara. Veremos que para ambos, la evolución y su divulgación entre las masas ha tenido influencias nefastas (sólo que cada uno opina sobre lo nocivo de movimientos contrarios) y ambos se pronuncian en torno a la muerte, como un término superable. Aunque su noción de hábito dista de ser igual, también se pueden establecer analogías. Sus puntos en común comienzan como absoluta disensión, aunque concluiremos con importantes confluencias.

Colin Wilson, biógrafo del intelectual dramaturgo, considera: «No hay nada en el libro de Bergson [La evolución creadora] que Shaw hubiera sido incapaz de escribir, y en el sentido más importante, la teoría de Bergson era más elemental que la de Shaw, por lo sobre enfático de la inhabilidad de la razón para atrapar los contornos flotantes de la vida, y eso tiende al anti-intelectualismo que D. H. Lawrence puso de moda más tarde» ${ }^{17}$. Wilson enjuicia a Shaw en otro aspecto que nos interesa: «En comparación, Shaw estaba sin influencia; ni uno de sus iguales intelectuales estaban de acuerdo con él» ${ }^{18}$.

11 Chesterton, G. K., George Bernard Shaw, Trad. José Méndez Herrera. Biblioteca de la Memoria. Renacimiento, Sevilla, 2010, p. 125.

12 Wilson, C., Bernard Shaw. A reassessment. The MacMillan Press Utd., London, 1981, p. 170.

13 Shaw, G. B., Hombre y superhombre. Introducción. A. C. Ward. Trad. Julio Broutá. Aguilar. 1966.

14 Grene, N., George Bernard Shaw. A critical view. The MacMillan Press, London, 1984, p. 161.

15 Chesterton, G. K., George Bernard Shaw, Trad. José Méndez Herrera. Biblioteca de la Memoria. Renacimiento, Sevilla, 2010, p. 176.

16 Ganz, A., George Bernard Shaw, The Macmillan Press, London, 1983, p. 50.

17 Wilson, C., Bernard Shaw. A reassessment. The MacMillan Press Utd, London, 1981, p. 170-171.

18 Ibid., p. 170-171. 


\section{EXPERIENCIA PSICOLÓGICA Y EXPERIENCIA INTERIOR}

En primer lugar, Shaw expresa que su teoría, es decir, la metafísica de la evolución, «se extrae de la experiencia personal de cada uno de nosotros ${ }^{19}$. Este punto de partida es perfectamente bergsoniano. Éste comienza La evolución creadora a partir de la experiencia personal, a partir de su propia idea de tiempo profundo y personal: la duración. Ahora veremos, en cambio, qué quiere decir Shaw con «experiencia personal». Shaw pregona en el Prefacio "el advenimiento del neo-lamarckismo ${ }^{20}$, lo cual, efectivamente, está directamente relacionado con la «experiencia personal». Este punto de partida es punto en armonía con Bergson por muy poco tiempo. Una vez atendemos que ambos pueden conceder al concepto de «experiencia personal», aparece la distancia: el psicologismo mal entendido es, según la doctrina bergsoniana, un error clave y antropomorfista del lamarckismo.

Debo comenzar esta confrontación explicitando que Henri Bergson, aunque es un declarado enemigo del mecanicismo (en último extremo, reducir el mundo de la vida al mundo sin espontaneidad creadora de la física, de lo atemporal, de la preeminencia de la causa sobre el efecto) no se puede decir que sea, como Shaw, neo-lamarckista. Aunque el neo-lamarckismo le haya inspirado en buena medida, Bergson no pretende que una jirafa individual haya legado los frutos germinales (según la terminología de la época) de su esfuerzo individual de alargar el cuello a sus descendientes. Para Shaw «la raza aprende lo mismo que aprendió el individuo ${ }^{21}$, y para Bergson esto es así, pero sólo en un sentido mucho más restringido.

Como veremos, para Bergson el neo-lamarckismo ha tenido una influencia social tan nefasta como para Shaw ha tenido el darwinismo. Para Bergson, la idea de que un hábito pueda ser heredado tiende a biologizar la cultura (meramente accidental), tiende a convertir las capas más superficiales de la naturaleza humana en artificialmente esenciales. En un orden no de moral, sino filosófico, el lamarckismo tiene dos aspectos: por un lado, está basado en el psicologicismo, en el finalismo de la actividad. Por otro, contiene una idea profunda de "esfuerzo» (l'effort), a la que debería prestarse mucha atención.

Hay que señalar también que las teorías de los neo-darwinistas constituyen una aportación también muy importante en el primer capítulo de La evolución creadora: las teorías del plasma germinativo, aquello susceptible de mutar y que forma parte de la esencia ensimismada y proto-sustancial de lo orgánico.

Para Bergson el neo-lamarckismo tenía un problema de conjunto: el deseo de un ser vivo sólo podía ser un deseo circunstancial proyectado hacia algo concreto de su medio, y, en cierta medida, está acotado por los límites de un medio determinado. La mentada y mítica jirafa lamarckiana se entiende sólo acotada en sentido por las hojas más altas de las acacias. Pero la novedad profunda de su modo de ser no se entiende, según el élan, por esta relación. Además, arrastra el lamarckismo, según Bergson, un serio problema en el terreno científico (lo cual era muy grave para el filósofo francés, contra lo que algunos han dicho): la adquisición de los caracteres adquiridos es extraordinariamente improbable. Bergson atiende más que Shaw a los datos que los biólogos han ido proporcionando. Según Bergson, en algunos casos, sólo se ha conseguido probar

19 Shaw, G. B., Back to Methuselah. A metabiological Pentateuch. Bernhard Tauchnitz, 1922. Collection of british and american authors. Vol. 4578, p. 18.

20 Ibid.

21 SHaw, G. B., Back to Methuselah. A metabiological Pentateuch. Bernhard Tauchnitz, Collection of british and american authors. Vol. 4578, 1922. Pág, 19. 
que los caracteres adquiridos que se han llegado a heredar han sido meramente «taras» infligidas científicamente o no, nunca caracteres añadidos. Se han mostrado cómo se heredan faltas, como pueda ser heredar el alcoholismo, pero no añadidos. Además, Bergson manifiesta sus dudas en torno a esto. Más adelante lo desarrollaré. En cierto sentido, el científico no puede «operar» con añadidos: no puede disfrazar de adquisición de carácter adquirido lo que en realidad es un carácter eliminado y su consiguiente malformación. En el debate sobre si el órgano crea la función, o la función al órgano, Shaw es mucho más atrevido, así como impreciso.

\section{El milagro automatizado del hábito}

La cuestión de la herencia de los caracteres adquiridos es central dentro del Capítulo I, de La evolución creadora: De la evolución de la vida: Mecanicismo y finalidad, de Bergson. Éste, hemos dicho, no concluye del lado del neo-lamarckismo de Charles-Édouard Brown-Séquard precisamente, sino más bien del lado de sus adversarios. Bergson se mantiene cerca de las ideas filosóficas que extrae del lamarckismo, y del finalismo en general, pero tiene sus puntos de acuerdo con el neo-darwinismo de Weismann y otros. En especial, en la cuestión de la herencia neo-darwiniana (que desarrollaría las vías del mutacionismo, con Hugo de Vries), pues ésta revelaba un sistema de continuidad, de heredabilidad y novedad que le resultaba más fiable y demostrado, y mucho más sugerente y «útil» para el bergsonismo. Así pues, ¿qué papel juega el hábito aquí? El hábito es el uso, la funcionalidad de esos «caracteres adquiridos». Si ellos se heredan, según el neo-lamarckismo, el hábito se hereda con ellos: el cuello de la jirafa, una vez heredado, comportaba la habitualidad de ese cuello, su uso. Recuérdese que el lamarckismo defiende la preeminencia de la función sobre el órgano.

En general, el rol del hábito en Bergson, y su irreconciliabilidad con la vida auténtica, es una cuestión que recorre, antes de sus investigaciones biológicas, sus primeras inquisiciones. En La risa. Ensayo sobre la significación de lo cómico (cuyo origen está en unas conferencias de 1884) habla del humor como un control de hábitos, las caricaturas indican el hábito adquirido de un modo "estúpido»22 (es decir, no social: mal adaptado). La «gracia» aparece contrapuesta a la inercia del hábito. El humor es el poso de gracia que queda en una tendencia inercial: es una estética, pero de rango bajo, una estética social. El último reducto de "gracia», el límite de la humanidad: el arte de los hábitos.

En su doctrina de la memoria, la «memoria habitual» aparece en el más superficial (o espacial) de los puestos. Explica en el Capítulo III de Materia y memoria (de 1894): «El hábito más bien que memoria, representa nuestra experiencia pasada, pero no evoca su imagen. La otra es la memoria verdadera ${ }^{23}$. Los hábitos son siempre superficiales y adquiridos: no se heredan meta-biológicamente jamás. «El recuerdo de la lección, en tanto aprendida de memoria, posee todos los caracteres del hábito. Como el hábito, se adquiere por la repetición de un mismo esfuerzo. Como el hábito, ha exigido primero la descomposición, luego la recomposición de la acción total. Como todo ejercicio habitual del cuerpo, en fin, es almacenado en un mecanismo que imprime un impulso inicial en un sistema cerrado de movimientos automáticos que se suceden en el mismo orden

22 Bergson, H., La risa. Ensayo sobre la significación de lo cómico. Trad. María Luisa Pérez Torres. Espasa, Madrid, 1973, p. 28.

23 Bergson, H., Materia y memoria. Trad. Pablo Ires, Cactus, Buenos Aires, 2006, p. 161. 
y ocupan el mismo tiempo» ${ }^{24}$. El hábito proviene del automatismo, es una inercia que se introduce en el cuerpo.

Sigamos con Bergson un poco más. Ahora volvamos a La evolución creadora, en polémica con el neo-lamarckismo: «Los caracteres adquiridos de que se trata son, las más de las veces, hábitos o efectos del hábito. Y es raro que en la base de un hábito contraído no se halle una aptitud natural. De manera que siempre puede uno preguntarse si lo que se transmite es el hábito adquirido por el soma del individuo o no será más bien una aptitud natural, anterior al hábito contraído; esta aptitud habría permanecido inherente al germen que el individuo lleva consigo, como ya era inherente al individuo y, por consiguiente, a su germen. Así, nada prueba que el topo se haya vuelto ciego porque ha adquirido el hábito de vivir bajo tierra; quizá sea porque los ojos del topo estaban en vías de atrofiarse por lo que éste tuvo que condenarse a la vida subterránea » ${ }^{25}$. Es decir, quizá un mero condicionamiento anterior, un brote espontáneo, delimite la trayectoria del ojo, ¿cómo puede saberse?

En cambio, según explica Shaw en el epígrafe duodécimo, Advenimiento de los neolamarckianos, el topo se introduce en lo subterráneo simplemente porque, de algún modo, «quiere». Y, según explica en el siguiente apartado, Cómo se heredan los conocimientos adquiridos, nadie sabe por qué. Quiere otro hábito.

En este punto, Bergson es infinitamente más sofisticado que el intelectual irlandés, y pretende «un impulso que pasa de germen a germen a través de los individuos» ${ }^{26}$, una capacidad de cambio o mutación «inherente a los gérmenes que ellos [los individuos] portan antes que su sola sustancia ${ }^{27}$. Pretende hibridar las teorías germinales del neo-darwinismo y de Weismann, y la filosofía general de Lamarck, del esfuerzo, de la insatisfacción, en definitiva, de todo lo viviente. La teoría de Shaw no atiende a un método riguroso. Según Shaw, "para el evolucionista todos los hábitos son adquiridos» ${ }^{28}$. En cierto modo, según él, todo lo que es, es hábito: no las meras acciones inteligentes. Por ejemplo, envejecer es un hábito ${ }^{29}$. «Las adquisiciones pueden ser asimiladas como cualificaciones congénitas ${ }^{30}$, el mismo proceso evolutivo es en sí mismo hereditario ${ }^{31}$.

Para Shaw hay unos hábitos (como el hábito de morirse, por ejemplo) que han arraigado en nuestra naturaleza. Otros, como, por ejemplo, los hábitos culturales históricos más arbitrarios y relativos, como el ajedrez, no se han hecho camino en nosotros, en nuestro cuerpo. No los hemos querido con la misma intensidad y no se han apropiado de nuestro organismo. Para Shaw somos los hábitos que hemos deseado, pero estamos abiertos a desear otros nuevos. Por eso, la destreza con la esgrima es algo que nuestra naturaleza no quiere necesariamente. Por eso decae entre padre e hijo descendiente. La aparición de nuestros dientes, hecho superviviente en las generaciones, es según Shaw, un hábito, pues son un querer de la naturaleza. En ese sentido, Shaw no establece una diferencia radical entre naturaleza y cultura, llamando a ambas «hábito», y a su producto «querer», «voluntad». Entre un ciclista y un artista hay, según él, una analogía posible con una ameba y un ser humano.

24 Ibíd., p. 91-92.

25 Bergson, H., L'évolution créatrice. PUF, Paris, 2006, p. 80.

26 Ibid., p. 86.

27 Ibid., p. 88.

28 SHaw, G. B., Back to Methuselah. A metabiological Pentateuch. Bernhard Tauchnitz, Collection of british and american authors. Vol. 4578,, 1922. p. 19.

29 Ibid., p. 20.

$30 \quad$ Ibid., p. 24.

$31 \quad$ Ibid., p. 24. 
En rigor, él toma la idea de que nuestra experiencia (nuestro querer cotidiano) es nuestra ventana al mundo, el querer del mundo: cualquiera de nuestras experiencias desiderativas y volitivas sirve como espejo del cosmos. No para Bergson, quien sólo consideraría unas concretas, muy privilegiadas, meta-psicológicas, para establecer esta analogía hombre/mundo evitando así el antropomorfismo. Así, para Bergson el planteamiento de Shaw podría representar un caso claro de antropomorfismo lamarckiano: reflejar la experiencia humana (el esfuerzo humano con respecto a fines preestablecidos) en el todo de la naturaleza. Esto se deriva en la teoría del tiempo de Bergson, a la que George Bernard Shaw no presta la menor atención.

Pero, cuando sólo se pueden apreciar como insalvables estas diferencias entre ambos, he de señalar que Bergson tiene también, como veremos, una visión del hábito como automatismo, como maquínico, inercial. Veamos cómo trata Bergson la cuestión del hábito.

Antes de nada, considero que, en sus puntos principales, Las dos fuentes de la moral y de la religión, de Bergson, constituye una prolongación antropológica sin fisuras de la doctrina expuesta en La evolución creadora, contra lo que opinan algunos comentadores. En su último libro, Las dos fuentes..., diez años, posterior al de Shaw, un anciano Bergson considera en torno al hábito y al ser humano que la naturaleza, es decir, la biología, es la que influye sobre la Historia humana, y nunca al revés. En ese sentido, la cultura, la Historia nunca podría transformar la esencia profunda de la humanidad. ¿Qué consecuencias tiene?

Leamos, antes de la aclaración pertinente, unas esclarecedoras líneas de Las dos fuentes de la moral y de la religión: «El señor Lévy-Bruhl extraerá la conclusión de que la inteligencia humana ha evolucionado: la lógica natural no habrá sido siempre la misma; la "mentalidad primitiva" corresponderá a una estructura fundamentalmente diferente, que la nuestra habrá suplantado y que no se encontrará hoy que en los retrasados (retardataires). Se afirma que los hábitos que el espíritu toma a través de los individuos a lo largo de los siglos han podido ser heredados, modificar la naturaleza y dar una nueva mentalidad a la especie. Nada más dudable» ${ }^{32}$. En este ensayo, como en La evolución creadora, se vuelve a hacer referencia a la dificultad de que el hijo contraiga un hábito creado de la nada por el padre frente a la mayor verosimilitud de que ambos contengan un principio germinal común que se desarrolla. El hábito es contingente, accidental: es fruto de un acomodo de un esfuerzo, de un cierto tipo de querer, sí, pero con su contexto. No es necesariamente un querer profundo. El hábito heredado es, dice Bergson, «accidental y excepcional» ${ }^{33}$. «Lo natural es hoy eso que ha sido siempre» («Le natural est donc aujourd'hui ce qu'il fut toujours») ${ }^{34}$, dice el francés, en referencia a la etnología evolutiva. Para él toda cultura será siempre un añadido. Y los añadidos no se heredan. Junto al etnólogo Lévy-Bruhl, Bergson criticará las estructuras sociales de Durkheim: tanto el uno como el otro, toman estructuras accesorias como esenciales a la naturaleza íntima del ser humano, que sólo es alterable a través de los «brotes» espontáneos de la vida. Allá donde biología y espíritu, vida y mística se dan la mano.

Efectivamente, la doctrina de Bergson es propiamente una doctrina (que toma de varias corrientes una forma compuesta pero sólida), y Shaw tiene una doctrina meta-biológica sólo impropiamente hablando, pues no nos dice por qué un hábito decae y porque otro arraiga en la naturaleza. Tampoco demarca el campo operativo de la meta-biología como tal.

32 Bergson, H., Les deux sources de la morale et de la religion. PUF, Paris, 2008, p. 107.

33 Ibíd., p. 168.

34 Bergson, H., Les deux sources de la morale et de la religion. PUF, Paris, 2008, p. 168. 
Pero tanto Shaw como Bergson tienen una visión común en este primer asunto: el hábito es una fuerza inercial, que se introduce en lo vivo. Y es una inercia que proviene de un esfuerzo. Es un milagro del esfuerzo pero ya normalizado hasta lo inconsciente. Es un salto del espíritu, convertido en ley material. Y frente a la inercia, está lo que la originó, su génesis. En un caso, el «élan». En otro, el «querer».

\section{ShaW CONTRA El DaRWinismo}

A modo de brevísimo excurso, subrayo el componente de provocación del ataque de Shaw al darwinismo, que es también un ataque a la anglofilia y, en general, a los valores dominantes de su sociedad. Como difunde a Schopenhauer, a Wagner, a Nietzsche, a Ibsen, él promociona a los metafísicos evolucionistas continentales anteriores a Darwin, que es la cumbre del orgullo intelectual victoriano: así, Shaw destaca a Buffon, a Lorenz, a Goethe, al Caballero de Lamarck frente a Charles Darwin o a Huxley. ¡Incluso su contendiente, August Weismann, biólogo alemán, es considerado por Shaw un hombre inteligente pero empujado a la imbecilidad por el darwinismo! También cuestiona las consideraciones del geólogo británico Charles Lyell, quien en Principios de geología amplía el tiempo de la tierra, lo cual funda un marco teórico propicio para el evolucionismo darwiniano, pues la amplitud temporal de la Tierra ralentiza el proceso evolutivo. La propuesta catastrofista de Shaw recuerda en esto a Spengler: ambos aseguraron que la evolución transcurrió en un periodo mucho más breve, sacudido de saltos, catástrofes y espontaneidad.

El darwinismo constituye el empobrecimiento intelectual del evolucionismo, según George Bernard Shaw. Es para él una nefasta superstición de la muchedumbre, la expansión de un pensamiento deshumanizado, que ha pretendido elevar el «método darwiniano» a gran teoría. El darwinismo había enfrentado los conceptos de Génesis y Evolución. ¡Tenemos que el darwinismo es un mal hábito! Es casi una redundancia en sí misma: el hábito automático del automatismo. Para Shaw, Weismann considera que todo es automático ${ }^{35}$. Bergson tiene una opinión mucho más positiva de Weismann. Pero tanto Shaw como Bergson, consideran que la Selección Circunstancial, o Selección Natural no es una respuesta positiva a la diferencia de especies humanas. Tan sólo es un procedimiento de aniquilación de algunas (de la mayor parte) de las especies vivientes. Explica la desaparición de los seres vivos, pero no sus apariciones. Ambos están de acuerdo en que el darwinismo no aborda el problema a fondo, si bien Bergson aprecia todas sus sofisticaciones formales y metódicas.

El discurso de Shaw, no especialmente riguroso, mezcla la historia de la biología con las cuestiones populares y políticas del momento. Pero su afán insistente en no distinguir la vida cultural y la vida del mundo biológico deriva en una cuestión, como veremos: la muerte, para la que, creo, Bergson sostiene un punto de vista no del todo disímil. Para Bergson la muerte biológica es, en el caso del hombre, un hecho superable. El hombre, el dueño de la técnica, puede tratar como accesorios, algunos aspectos de su determinación orgánica heredada desde el inicio de los tiempos. Sostengo que la confrontación con Shaw nos muestra un punto clave, y no por cierto derivado, en la teoría antropológica de Bergson.

Pero continuemos. El darwinismo tiene un correlato ideológico, según el dramaturgo, como he señalado. «Los socialistas estuvieron envalentonados por la insistencia de

35 SHaw, G. B., Back to Methuselah. A metabiological Pentateuch. Bernhard Tauchnitz. Collection of british and american authors. Vol. 4578, 1922. p. 40. 
Darwin en la influencia del medio ambiente» ${ }^{36}$, explica. Pero, por cierto, el neo-darwinismo no señala la causa de la variación entre especies provenga directamente del medio ambiente, como señala Shaw, aunque la condicione. Bergson señala al menos que eso es la ortogénesis, de Theodor Eimer. ${ }^{37}$

Por otro lado, Shaw, como Schopenhauer, defensor de los animales, hace aparecer la figura del «cirujano darwinista» ${ }^{38}$. Habla Shaw de la crueldad que demuestra el insensato Weismann intentando comprobar que, por cortar los rabos a los ratones y por mostrar, subsiguientemente, que aquéllos no se perpetúan sin rabo, ha demostrado algo. Aunque Shaw olvida que la provocación experimental de trastornos tróficos provocados en animales fue una práctica también extensible al bando de evolucionistas neolamarckianos. Precisamente, para sostener lo contrario que Weismann: el lector de $L a$ evolución creadora sabe que Nouvelles recherches sur l'épilpesie due à certaines lesions de la moelle épinière et des nerfs rachidiens, de Brown-Séquard, o Transmission expérimentale aux descendants de lésions développées chez les ascendants, de Charrin, Delamare y Moussu $^{39}$, forman parte de la exótica y extraña bibliografía anotada de Bergson. Son informes neo-lamarckianos donde se explican las consecuencias de taras provocadas por científicos a conejillos de indias. Se trata de seccionamientos del nervio ciático y la médula espinal, o de lesiones en el hígado o riñón en ejemplares en periodo de gestación.

Dejando de lado estas últimas particularidades, Shaw señala que la biología mecanicista que él combate había tapiado la vía hacia la meta-biología de finales del siglo xviII. Además, condenando al ser humano dentro de una tupida red causal impersonal, se había acabado con la idea de genio espontáneo. "Jesús automático, y Shakespeare automático ${ }^{40}$. En parte, según Shaw, el desastre de la Primera Guerra Mundial se dio por aquellas nuevas ideas de la segunda mitad de siglo XIX. «A causa de la selección confiaron en que era puramente una selección circunstancial oportunista» ${ }^{41}$.

La nueva sociedad no privilegiaba el esfuerzo personal, ni, en general, las fuerzas del espíritu. La falsa idea de pasividad evolutiva había refrenado el carácter creador del hombre. Lo había conducido a la mediocridad y, finalmente, a la destrucción. Shaw, en sus accesos de libelista, habla de cobardía y de irresponsabilidad. El automatismo del hábito no renovado (lo anquilosado por excelencia) se había expandido por el mundo, cuando, según él, el nuevo evolucionismo pre-darwinista preparaba un debate intelectual muy superior, lleno de grandes valores creadores activos, de nuevas propuestas de nuevo querer.

\section{BERGSON CRITICA Y AMPLÍA EL LAMARCKISMO}

Como he apuntado, la cuestión de la herencia de los caracteres adquiridos, ocupa buena parte del primer capítulo de La evolución creadora. En especial, se aborda en el epígrafe La herencia de lo adquirido (L’hérédité de l'acquis) ${ }^{42}$, donde Bergson acaba

36 Ibid., p. 48.

37 Bergson, H., L'évolution créatrice. Presses Universitaires de France, Paris, 2006, pp. 71-76.

38 Shaw, G. B., Back to Methuselah. A metabiological Pentateuch. Bernhard Tauchnitz, Collection of british and american authors. Vol. 4578, 1922, pp. 41, 43, 45.

39 Bergson, H., L'évolution créatrice, Presses Universitaires de France, Paris, 2006, pp. 81-82.

40 Shaw, G. B., Back to Methuselah. A metabiological Pentateuch. Bernhard Tauchnitz, Collection of british and american authors. Vol. 4578, 1922, p. 40.

41 Ibid., p. 57.

42 Bergson, H., L'évolution créatrice. PUF, Paris, 2006., pp, 77-86. 
dando la razón a los neo-darwinianos ${ }^{43}$. La teoría del plasma germinativo de Weismann le permite atisbar la idea de un fondo, de un sustrato molecular más allá de los individuos concretos. La materia viva: su mutación (de acuerdo con los parámetros de De Vries), esto es, su cambio espontáneo, no se entiende a partir de la circunstancia o hábitat, sino más bien a partir de la espontaneidad. Sin finalidad, es decir, sin un programa que se realiza, como toda acción humana, con respecto a un plan atemporal, es decir, eterno, sin duración real, intelectualmente predeterminado. El lamarckismo tiene pues, como finalismo que es, una estructura intelectualista de la que debería desembarazarse.

Tenemos una nueva disensión entre Shaw y Bergson. Como hemos visto, en su tratado antropológico, después de la Primera Guerra Mundial (y ocho antes de la Segunda) y después de haber ocupado un cargo de responsabilidad en la Sociedad de Naciones, Henri Bergson señala entre los culpables de la enfermedad de su tiempo al neo-lamarckismo, curiosamente. No culpabiliza, como Shaw, al neo-darwinismo. En Las dos formas de la moral y de la religión, explica que el lamarckismo, y en especial la noción de la heredabilidad de los caracteres adquiridos ha hecho «mucho mal»4${ }^{44}$ Lo dice en el capítulo IV, último de este libro, que es también una proclama a favor de la abolición de la guerra y la prolongación de la vida hacia un más allá. Hacia un superhombre. Y, famosamente, habla del cosmos como una fábrica de hacer dioses.

En último extremo, Bergson situaría a Shaw en un ámbito de verdadera falta de libertad. De acuerdo, podría decir, su filosofía habla de una fuerza, como la «fuerza creadora» que Shaw expone primero en Hombre y superhombre y después en Vuelta a Matusalén, tomada en buena medida de la "Voluntad de poder», de Nietzsche (lector, por cierto, de Lamarck). Pero Bergson reniega del lamarckismo pues, en el fondo, comporta que los individuos (una jirafa, un topo) sólo pueden desear cosas individuales. Y esas cosas individuales deseadas les preceden. La carrera del deseo imposible de Lamarck dibuja un mapa donde todos los seres vivos pretenden una perfecta adaptación al medio. De modo que el medio demarca el punto, el estado de perfección más allá del progreso de la duración. Según el pensador francés, el lamarckismo es un tipo de finalismo, pero amarrado a cuestiones biológicas: es la trasposición biologicista de la doctrina filosófica teleológica clásica desde Aristóteles. El medio donde el ser vivo nace es su fin, el marco donde sus tendencias se comprenden y se realizan. Bergson es más radical aquí.

Bergson postula una creación de formas limitada por la Selección Natural, pero en cierto modo, un curso ensimismado de herencia y creación de novedad. La vida crea para sí misma, no para el medio. No para una mejor adaptación al medio. Bergson no defiende un paradigma armónico, como, después de tanto aparente dramatismo, termina mostrándose el lamarckismo. Por eso, repito, hibrida las teorías neo-lamarckianas y las neo-darwinistas, aportándoles una nueva dirección y sentido. Un sentido plenamente artístico, irreductible a concepto o fin. Frente a la exterioridad somática que propugna, en todo caso, el lamarckismo metafísico, el neo-darwinismo de Weismann (celebrado por Bergson, lamentado por Shaw) concibe una interioridad orgánica, el germen, el protoplasma germinativo, que no opera en función de esto o aquello, sino porque sí. Sin fin pre-definido. Bergson establece un segundo momento subsiguiente donde lo creado se somete al rigor racional del mundo somático. Pero también señala que éste no sirve como causa profunda.

El finalismo lamarckiano cae, según Bergson, en el antropomorfismo. De tal modo, el anterior epígrafe Experiencia psicológica y experiencia interior, quería resaltar que

43 Ibid., p. 86.

44 Bergson, H., Les deux sources de la morale et de la religion. PUF, Paris, 2008, p. 321. 
Shaw toma una experiencia general de la vida, una experiencia de actividad, y la extrapola a la Naturaleza misma con cierta facilidad. Bergson propugna una intuición profunda, no una experiencia «cualquiera», sino una experiencia privilegiada de la propia duración para conocer y simpatizar con el mundo entero. La propuesta de nuestro «querer» habitual (Shaw) no deja de ser un tosco remedo.

Por eso es la idea de experiencia de duración, de tiempo interior bergsoniano es más restringida que la de Shaw, que aplica la idea de «deseo» o «voluntad» a todo lo existente. Sobre todo, la postura de Bergson se vuelve mucho más meticulosa en su relación con «los hechos científicos», lo cual, sin duda, no pasó desapercibido para Bertrand Russell cuando enjuicia el "estilo del prefacio de Matusalén». Hay que apuntar también que la experiencia de la embriología, de fulgurante desarrollo a principios de siglo xx, admiró por igual a Bergson y a Shaw, como a Driesch y a von Uexküll. Se demostraba que el feto humano atravesaba las previas fases biológicas en cuestión de días hasta llegar al estadio humano. Quedaba patente que el hombre era un punto privilegiado, una atalaya orgánica, para entender la evolución.

Pero en especial, más allá de los datos de la ciencia, Shaw y Bergson mostraron atención no por cualquier hombre, sino por el hombre genial. Su actividad también expresa, de modo privilegiado, la creación de nuevas formas de la naturaleza. El arte también reúne amistosamente a los dos ensayistas. Si Shaw repasa, acaso con torpeza, los datos de la naturaleza, y, acaso sin rigor, la noción del hábito, con o sin torpeza, sin o con rigor, el dramaturgo se encuentra perfectamente con Bergson reivindicando el arte como gran creación humana y natural.

Bergson, quien extrañamente nunca publicó un texto sobre estética ${ }^{45}$, nunca dejó de hablar sobre ella. La refiere en el punto culminar de todos y cada uno de sus discursos. Shaw consideró irrefutable, en último extremo, el mecanicismo, y al mismo tiempo lo despreció. La idea de que todo está determinado le parecía un baluarte tan sólido como ninguneable. Para Bergson, la propuesta del finalismo (no el lamarckiano, pero sí sus ampliaciones cósmicas) le parecían ampliables, pero imposibles de transgredir del todo. Frente al mundo sin novedad que Shaw creía invencible y dañino (pero que Bergson consideró vapuleado por sus argumentos), y frente al finalismo (donde Bergson sintió que sus argumentos languidecían levemente, en parte "ampliando», y no destruyendo la noción), ambos prefirieron pensar en la naturaleza como un genio creador. Jesucristo y Shakespeare no podían ser, ni automáticos, ni obreros inteligentes. El arte es para Shaw y para Bergson el gran paradigma de sanidad y de humanidad proyectada a un más allá. Conocían la fuerza del argumento de sus respectivos enemigos (para Shaw, el mecanicismo, para Bergson, que creyó derrotar plenamente al mecanicismo, se trataba del finalismo), y se entregaban a la elocuencia del caso irreductible de la estética.

\section{LA MUERTE ANTROPOLÓGICA: BIOLOGÍA Y HABITUALIDAD}

«Lo que significa que si el hombre no va a ser salvado, el hombre debe salvarse a sí mismo ${ }^{46}$, sentencia Shaw. Como hemos dicho, la muerte masiva de la guerra lleva su mirada a un futuro cargado de una ilusión: un más allá, una prolongación de la vida más allá de

45 Dresden, S., Les idées esthétiques de Bergson. Les études bergsoniennes (53-75). IV. PUF, Paris, p. 56.

46 Shaw, G. B., Back to Methuselah. A metabiological Pentateuch. Bernhard Tauchnitz. Collection of british and american authors. Vol. 4578, 1922, p. 14. 
la defunción natural. ¿Se puede alargar la vida indefinidamente? ¿Es accidental la muerte en los seres humanos, nacidos íntimamente, según él, para perdurar? Las tortugas y los loros viven más, dice, porque «quieren vivir más». Una longevidad extrema, como vemos en sus dramas ilustrativos Hasta donde alcanza el pensamiento y La cosa funciona, sería factible, si apareciera ese deseo de la especie. En este tramo, el discurso de Shaw se vuelve inconcreto. Según Shaw, la muerte es siempre accidental ${ }^{47}$. En la medida en que algo natural pueda ser accidental para Shaw, pues ya mostré que los hábitos son para él naturales como el crecimiento del cuerpo, y por tanto, no accidentales, como diría Bergson. En el discurso de Shaw advertimos una accidentalidad de grado: lo más accidental es lo que deja de usarse.

Bergson se mantiene notablemente más cauto: el órgano y la función son simultáneos, no precede el uno al otro. Pero la «función» que en el capítulo IV de Las dos fuentes de la moral y la religión: Mecánica y mística (Observaciones finales) otorga al esfuerzo es aquella de hacer dioses ${ }^{48}$, es decir, nuevos núcleos de creación. Apunta claramente a un más allá de la humanidad. En el final del capítulo III de La evolución creadora el autor ya apuntó a ese más allá de la humanidad, con una coda impresionante. La humanidad es una armada que carga capaz sortear resistencias y obstáculo, «quizá incluso la muerte» ${ }^{49}$. Insisto en que, al menos en este aspecto, Las dos fuentes de la moral y de la religión no contiene ninguna contradicción con La evolución creadora. Es más, la continúa perfectamente.

Se trata de lo que David Lapoujade considera que es «sobrepasar la condición humana ${ }^{50}$. Dice Lapoujade: «Si, a pesar de todo, el hombre conserva un lugar privilegiado en el bergsonismo, es porque a su vez es el único en poder saltar por encima de su inteligencia y recorrer, como pensamiento, todos los niveles de la realidad que lo desbordan $»^{51}$.

Finalmente, Bergson y Shaw, pese a sus importantísimas distancias en torno al hábito y a la naturaleza de la Vida, y de la experiencia, profetizan un superhombre, más allá de la muerte. En el caso de Bergson se habla de una alianza entre la técnica y el hombre, pero porque el hombre tiene en sí un impulso de vida que de duración ininterrumpida, nunca quebrantada del todo. La muerte bergsoniana tiene un estatus un tanto híbrido: de orden orgánico, y no habitual, sino germinal. Pero superable, quizá por el empeño de la humanidad entera en transgredir sus bordes. El comentador de la obra de Shaw, Arthur Ganz explica: «Pero incluso la especulación crucial de que la vida debe sobrepasar la muerte, que Shaw comparte con Bergson, es presentada por Shaw como la superación de un hábito inútil, una cuestión que lo relaciona concluyentemente con Butler» ${ }^{52}$.

Por fin, podemos preguntarnos, ¿es la muerte, entendida en su sentido antropológico, es decir, la muerte comprendida como límite absoluto, la muerte humana, un hábito de la humanidad o una determinación del mundo viviente? Es decir, ¿se ha apropiado la inercia del hábito de ese radical de la experiencia vital?

Parece que Bergson ensaya con el protoplasma germinativo del neo-darwinismo, una idea de élan viviente ininterrumpido desde sus inicios, una fuerza sin verdadero término. Shaw, sin una idea elaborada del desarrollo desde los albores, defiende

47 Shaw, G. B., Back to Methuselah. A metabiological Pentateuch. Bernhard Tauchnitz. Collection of british and american authors. Vol. 4578, 1922, p. 19.

48 Bergson, H., Les deux sources de la morale et de la religion. PUF, Paris, 2008. «Ella [la Humanidad] debe preguntarse si quiere vivir solamente, o realizar, además, el esfuerzo necesario para que se cumpla, sobre nuestro planeta refractario, la función esencial del universo, que es una máquina de hacer dioses». «machine à faire des dieux», p. 338.

49 Bergson, H., Lévolution créatrice. PUF, Paris, 2006, p. 271.

50 Lapoujade, D., Potencias del tiempo. Trad. Pablo Ires. Cactus, Buenos Aires, 2010, p. 218.

51 Ibid., p. 91.

52 Ganz, A., George Bernard Shaw. The Macmillan Press Ltd, London, 1983, p. 50. 
simplemente una voluntad de crecer de todo lo viviente. Una fuerza particularmente latente en el ser humano.

Así pues, ¿no es para Bergson, en parte, si esto es cierto y la muerte es sólo un obstáculo, un principio a medias entre la heredad biológica y el hábito, en principio accidental? ¿Qué lugar ocupa? Por un lado, el ser humano ha heredado de sus ancestros individuales, una mortalidad natural. Por otro, ha heredado, del curso que media entre viviente y viviente, un principio de continuidad sin detención.

Parece que el ser humano, según Bergson, en cuanto reconoce la muerte como un obstáculo, la antropomorfiza (pero esta vez legítimamente), la convierte en obstáculo, en accidente. Su mera ansia de vida es significativa, parece. Pero esa ansia de vida no ha sido directamente heredada del mundo animal, aunque sí del todo de lo viviente. Parece que la más tosca relación entre naturaleza y hábito antropológico humano de Shaw también tiene un punto de confluencia con Bergson en este punto, en este límite que la muerte supone para el hombre: ese hábito al que no está habituado. Dejemos de lado la posible utilidad (lo cual redunda en su adaptación correcta en el mundo) que se pueda extraer, en conjunto, para la humanidad, el hecho de que haya muerte. La muerte es acaso una adaptación del hombre como especie al mundo (pues como especie, no tendría cabida), pero, como individuo, está formalmente inadaptado orgánicamente a la muerte. Por eso la trata como hábito, como artificio, no natural. La muerte es organismo, por un lado, y una base de la cultura por el otro. Es una heredad a la que nunca cabe habituarse, porque trasciende el campo del horizonte de los hábitos. A este horizonte futurizo apunta la humanidad tanto en Shaw como en Bergson.

En George Bernard Shaw, Chesterton, explica: «Lo cierto es que los espíritus débiles viven, naturalmente, en el futuro, porque éste no tiene rasgos característicos; y la tarea es fácil, ya que podéis imaginároslo como gustéis. La época próxima está en blanco [...]» $\aleph^{53}$. El polemista inglés y biógrafo de Shaw explica que «el gran defecto de esta hermosa inteligencia es el no poder captar y gozar de las cosas comúnmente llamadas convención y tradición $»^{54}$. Y añade Chesterton: «Las convenciones no son cosa muerta jamás...» $»^{55}$. Chesterton supone un perfecto contrapunto a los dos protagonistas de este artículo.

Aunque el padre Tonquédec señala que «igual que la filosofía de Bergson busca romper los moldes donde las preocupaciones utilitarias de la vida han deformado las intuiciones primitivas, así hace el artista según Chesterton ${ }^{56}$, la mirada de Bergson nunca atribuye a la convención el carácter primigenio. Chesterton aboga por una filosofía de los contornos concretos, frente a las abstracciones categóricas. Bergson no contrapone estos polos, sino, más bien, acusa a lo concreto de derivado espacializante de lo abstracto. Volviendo a la convención práctica determinada: tanto en el hombre primitivo como en el actual, hablamos de adquisiciones leves, accidentales.

Según Bergson, el hombre ha heredado algo extraño en lo que a su vida concierne, un principio de contingencia práctica: el suyo es el hábito de tener hábitos. Los místicos, por ejemplo, rescatan algo de lo originario. La mística proviene de la naturaleza biológica, es decir, profunda y espiritual del hombre, y no puede entrar en el mundo de los hábitos. El misticismo no se puede normalizar. No hay convención en él. Según Shaw las religiones expresan también verdades originarias: verdades de creación. Por eso, Shakespeare o

53 Chesterton, G. K., George Bernard Shaw, Trad. José Méndez Herrera. Biblioteca de la Memoria. Renacimiento, Sevilla, 2010, p. 170.

54 Ibid., p. 132.

55 Ibíd.

56 Tonquédec, J. De., G. K. Chesterton. Ses idées et son caractère. Paris Nouvelle Librairie Nationale. Paris, MCMXX, p. 81. 
Goethe proponen una religión. Según estima Shaw, parece que sin duda, Goethe está un escalón más arriba en lo que a la religión de la evolución creadora se refiere.

El artista es tan fundamental para Bergson como para Chesterton y Shaw. Su arte es un culmen en la teoría del conocimiento. Son intuicionistas. Por volver mentar al tecer contendiente, Chesterton considera que la verdad del arte no puede alejarse del misterio de la convención espacial: no son mundos enfrentados. Por eso la muerte es un misterio constitutivo para entender el pasado, nunca un obstáculo para expandir el futuro. Shaw es neo-lamarckiano y anti-darwiniano, Bergson ensaya un añadido a la síntesis de ambas posturas. Shaw convierte la cultura en naturaleza evolucionante y Bergson distingue rigurosamente la una de la otra, ambos ven en las doctrinas evolucionistas un carácter de «mal hábito» en sí mismas, y ambos consideran a sus contendientes, en último extremo, como imbatibles. Shaw apela a un concepto general, tomado del voluntarismo pero aplicado a la evolución: la muerte es mudable como cualquier convención. En sus accesos más «proféticos», Henri Bergson llega, en torno a la muerte, a un concepto límite, bastante misterioso y velado, entre el mundo de la naturaleza y el mundo del hombre. No sabemos, con certeza, hasta qué punto podríamos seguir hablando de hombre y humanidad más allá de esta línea de horizonte, al fin de este recorrido por las periferias del bergsonismo. Determinando el grado de accidental o de esencial que el atributo de mortandad tiene en lo humano, en su naturaleza, podríamos alcanzar a saber si es la muerte el obstáculo, o, en sí misma, la humanidad, como detención de un impulso imponderable, mucho más ambicioso.

\section{BibliogRAFÍA}

Bergson, H., El pensamiento y lo moviente. Trad. Heliodoro García. Espasa, Madrid, 1971.

Bergson, H., La risa. Ensayo sobre la significación de lo cómico. Trad. María Luisa Pérez Torres. Espasa, Madrid, 1973.

Bergson, H., L'évolution créatrice. PUF, Paris, 2006.

Bergson, H., Les deux sources de la morale et de la religion. PUF, Paris, 2008.

Bergson, H., Materia y memoria. Trad. Pablo Ires, Cactus, Buenos Aires, 2006.

Chesterton, G. K., George Bernard Shaw, Trad. José Méndez Herrera. Biblioteca de la Memoria. Renacimiento, Sevilla, 2010.

Delattre, F., «Henri Bergson et l'Anglaterre», in Les études bergsoniennes. Vol. II, PUF, Paris, 1949.

DRESDEN, S., "Les idées esthétiques de Bergson». Les études bergsoniennes (53-75). IV. PUF, Paris, 1968.

Ganz, A., George Bernard Shaw. The Macmillan Press Ltd-P. 50. London, 1983.

Grene, N., George Bernard Shaw. A critical view. The MacMillan Press, London, 1984.

Lapoujade, D., Potencias del tiempo. Trad. Pablo Ires. Cactus, Buenos Aires, 2010.

Russell, B., Historia de la Filosofía Occidental. Trad. Julio Gómez de la Serna y Antonio Dorta. Austral, Madrid, 1999.

Russell, B., Retratos de memoria y otros ensayos. M. Aguilar Editor. Manuel Suárez, Buenos Aires. 1960.

SHaw, G. B., Back to Methuselah. A metabiological Pentateuch. Bernhard Tauchnitz. Collection of british and american authors. Vol. 4578. 1922.

SHaw, G. B., Hombre y superhombre. Introducción. A. C. Ward. Trad. Julio Broutá. Aguilar. 1966.

Soulez, P.; Worms, F., Bergson. Biographie. PUF, Paris, 2002.

Tonouédec, J. De. G. K. Chesterton., Ses idées et son caractère. Paris Nouvelle Librairie Nationale, Paris, MCMXX.

WiLson, C., Bernard Shaw. A reassessment. The MacMillan Press Utd, London, 1981.

Universidad Diego Portales.

Programa de Doctorado en Filosofía Udp-Leiden

Álvaro Cortina Urdampilleta

alvarocortina@hotmail.com

[Artículo aprobado para publicación en noviembre de 2013] 